\title{
Resultados das Intervenções Coronárias Percutâneas Primárias Realizadas nos Horários Diurno e Noturno
}

\author{
Cristiano O. Cardoso, Daniela Dalla Lana, Grasiele Bess, Juliana Cañedo Sebben, Eduardo Mattos, \\ Felipe A. Baldissera, Rogério Sarmento-Leite, Alexandre S. Quadros, Carlos Antônio Mascia Gottschall
}

\begin{abstract}
RESUMO
Introdução: Estudos demonstram que a intervenção coronária percutânea primária realizada fora do horário de rotina está relacionada a pior prognóstico. Nosso objetivo foi avaliar os desfechos da intervenção coronária percutânea primária realizada nos períodos diurno e noturno em um centro cardiológico de referência. Métodos: Estudo de coorte prospectivo, que incluiu 1.108 pacientes consecutivamente atendidos por infarto agudo do miocárdio com supradesnivelamento do segmento ST, divididos nos grupos intervenção coronária percutânea primária diurna (se realizada entre 8 e 20 horas) e intervenção coronária percutânea primária noturna (se realizada entre 20 e 8 horas). Resultados: Incluímos 680 pacientes no grupo diurno e 428 no grupo noturno. As características basais referentes ao perfil demográfico, fatores de risco e classificação Killip foram semelhantes em ambos os grupos, porém o tempo porta-balão foi significativamente maior no grupo noturno $(84 \pm 66$ minutos vs. $102 \pm 98$ minutos; $p<0,01)$. Vasos culpados, e fluxos TIMI pré e pós-procedimento não foram diferentes entre os grupos. Não encontramos diferenças significantes em relação à mortalidade hospitalar $(7,6 \%$ vs. $10,2 \% ; p=0,16)$, trombose de stent $(2,8 \%$ vs. $2,4 \% ; p=0,69)$ ou presença de sangramento maior $(1,9 \%$ vs. $2,1 \% ; p=0,50)$. Em 1 ano, a mortalidade também foi semelhante $(9,5 \%$ vs. $12,6 \% ; p=0,12)$. O principal preditor de mortalidade em 1 ano foi a classe III/IV de Killip (OR = 10,02; IC 95\% 5,8-17,1; $p<0,01)$. Conclusões: Pacientes com infarto agudo do miocárdio apresentam taxas de desfechos clínicos semelhantes, independentemente do horário de realização da intervenção coronária percutânea primária. No entanto, o tempo porta-balão é significativamente maior nos pacientes tratados entre 20 e 8 horas.
\end{abstract}

DESCRITORES: Infarto do miocárdio. Intervenção coronária percutânea. Reperfusão miocárdica.

\section{ABSTRACT}

Results of Primary Percutaneous Coronary Interventions Performed During On- and Off-Hours

Background: Previous studies have shown that off-hours primary percutaneous coronary interventions are related to a worse prognosis. The objective of this study was to evaluate the outcomes of patients undergoing on- and off-hours primary percutaneous coronary interventions performed at a reference cardiology center. Methods: Prospective cohort study, including 1,108 consecutive patients with ST elevation myocardial infarction divided into primary percutaneous coronary intervention performed during regular working hours group (on-hours: 8:00 am to $8: 00 \mathrm{pm}$ ) and primary percutaneous coronary intervention during nonregular working hours group (off-hours: 8:00 pm to 8:00 am). Results: The sample included 680 patients in the on-hours group and 428 in the off-hours group. Baseline demographic data, risk factors and Killip classification were similar in both groups, however door-to-balloon time was significantly longer in the off-hours group $(84 \pm 66$ minutes vs. $102 \pm 98$ minutes; $p<0.01)$. Culprit vessels, pre- and post-procedure TIMI flows were not different between groups. There were no significant differences for in-hospital mortality (7.6\% vs. $10.2 \% ; p=0.16)$, stent thrombosis $(2.8 \%$ vs. $2.4 \%$; $p=0.69)$ or major bleeding (1.9\% vs. $2.1 \% ; p=0.50)$. One-year mortality was also similar (9.5\% vs. $12.6 \% ; p=0.12)$. The main predictor of mortality at 1 year was Killip III/IV (OR, 10.02; 95\% Cl, 5.8-17.1; $p<0.01)$. Conclusions: Patients with myocardial infarction have similar in-hospital clinical outcomes regardless of the time primary percutaneous coronary intervention is performed. However, door-to-balloon time is significantly longer in patients treated during off-hours.

DESCRIPTORS: Myocardial infarction. Percutaneous coronary intervention. Myocardial reperfusion.
Instituto de Cardiologia do Rio Grande do Sul, Fundação Universitária de Cardiologia, Porto Alegre, RS, Brasil.
Correspondência: Cristiano O. Cardoso. Rua Francisco Petuco, 340/805 Boa Vista - CEP 90520-620 - Porto Alegre, RS, Brasil.

E-mail:cro_cardoso@yahoo.com.br

Recebido em: 10/12/2013 • Aceito em: 25/2/2014 
A intervenção coronária percutânea primária (ICPp) mostrou-se superior à terapia trombolítica como método de reperfusão no infarto agudo do miocárdio com supradesnivelamento do segmento ST (IAMCSST). ${ }^{1-3}$ Além de restaurar o fluxo coronariano em mais de $90 \%$ dos casos, ela é capaz de aumentar a sobrevida, e de reduzir as taxas de reinfarto e de acidente vascular encefálico relacionados à trombólise química. ${ }^{4-9}$ Frente a esses benefícios, a ICPp tem indicação classe I para tratamento do IAM nas primeiras 12 horas de evolução, quando disponível em período menor que 2 horas do primeiro contato médico e realizada em centros capacitados. ${ }^{7,10,11}$

Com a relação à aplicabilidade da ICPp, diversos estudos têm sugerido que o tempo porta-balão e que a mortalidade podem ser elevados no período noturno, comparativamente ao horário diurno..$^{12-15}$ No entanto, dados avaliando essa variável têm sido pouco relatados na literatura nacional. ${ }^{16}$ Por conseguinte, foi objetivo deste trabalho avaliar os desfechos da ICPp realizada no horário de rotina (diurno) e no plantão (noturno) em um centro cardiológico de referência.

\section{MÉTODOS}

\section{Delineamento}

Estudo observacional com coleta de dados prospectiva.

\section{Amostra}

Pacientes com diagnóstico de IAM e submetidos à ICPp em um centro terciário de cardiologia intervencionista foram incluídos neste estudo. Utilizaram-se, como critérios de inclusão, os diagnósticos clínico e eletrocardiográfico de IAM, e a indicação de tratamento por ICPp pelo médico assistente. Os critérios de exclusão foram IAM > 12 horas de evolução (exceto aqueles em choque cardiogênico), menores de 18 anos ou recusa em participar do estudo. Todos os pacientes foram entrevistados na admissão hospitalar e assinaram termo de consentimento livre e informado, previamente aprovado pelo comitê de ética em pesquisa local.

\section{Procedimento de intervenção coronária percutânea primária}

Os pacientes receberam dose de ataque de 300 a 500 mg de ácido acetilsalicílico e 300 a 600 mg de clopidogrel. O uso de morfina, heparina endovenosa, nitrato sublingual/endovenoso ou betabloqueador ficava a critério do médico plantonista. O paciente era conduzido para a realização do procedimento assim que a sala de procedimentos estivesse disponível. Em nossa instituição, existe uma equipe de enfermagem de plantão no setor de cardiologia invasiva 24 horas do dia, 7 dias por semana. O cardiologista intervencionista é chamado pelo médico da emergência nos casos de ICPp, e a rotina da instituição requer que ele esteja presente 30 minutos após o chamado.
A ICPp foi realizada conforme preconizado por diretriz, ${ }^{7}$ sendo a via de acesso definida pelo intervencionista. Todos os pacientes receberam heparina endovenosa na dose 60 a 100 U/kg. Pré-dilatação, administração de inibidores de glicoproteína Ilb/IIla, tromboaspiração e implante de stent foram realizados de acordo com o julgamento do operador. O uso do balão intra-aórtico ficou restrito aos pacientes com choque cardiogênico.

\section{Definições}

O IAM foi definido como a: (1) presença de dor em repouso associada a supradesnivelamento do segmento ST de $1 \mathrm{~mm}$, em pelo menos duas derivações contíguas ou (2) presença de dor em pacientes com bloqueio de ramo esquerdo, presumivelmente novo. Os IAMs foram classificados como: tipo 1 se IAM espontâneo relacionado a evento coronariano primário; tipo 2 se IAM secundário à isquemia por aumento da demanda ou redução da oferta de oxigênio; tipo 3 se morte cardíaca; tipo 4a se IAM relacionado à ICP; tipo $4 \mathrm{~b}$ se IAM por trombose de stent documentada à angiografia; e tipo 5 se IAM relacionado à cirurgia de revascularização miocárdica. ${ }^{17}$

O tempo porta-balão foi definido como o período entre a admissão hospitalar e a primeira insuflação do cateter balão ou liberação do stent (nos casos de stent direto).

Considerou-se como sangramento menor a presença de hemorragia clinicamente evidente como queda de hemoglobina entre 3 e $5 \mathrm{~g} / \mathrm{dL}$ ou hematócrito entre 9 e $15 \%$. Sangramento maior foi definido como a ocorrência de hemorragia clinicamente evidente com queda dos níveis de hemoglobina > $5 \mathrm{~g} / \mathrm{dL}$ ou hematócrito > 15\% ou desenvolvimento de acidente vascular encefálico hemorrágico.

Classificou-se como trombose do stent a oclusão súbita do vaso tratado, confirmada por angiografia, durante a fase hospitalar.

\section{Seguimento clínico}

Os pacientes foram acompanhados no período hospitalar por um dos investigadores do estudo e os desfechos avaliados foram: mortalidade, acidente vascular encefálico, trombose de stent, sangramento maior ou menor, e insuficiência renal com necessidade de diálise. Seguimento clínico em 30 dias e em 1 ano foi obtido por visitas ambulatoriais ou contato telefônico.

\section{Análise estatística}

Os dados foram prospectivamente armazenados em um banco de dados ACCESS e analisados pelo Statistical Package for the Social Sciences (SPSS) 18.0 para Windows. As variáveis categóricas foram descritas como números absolutos e porcentuais, e comparadas com o teste qui-quadrado. As variáveis contínuas foram 
apresentadas como média e desvio padrão, e comparadas com o teste $t$. O teste de Mann-Whitney foi utilizado em casos em que as variáveis contínuas não apresentaram distribuição normal. Análise de regressão logística múltipla foi utilizada para identificar preditores independentes de mortalidade. Foi considerado como de significância estatística um valor de $p$ bicaudal $<0,05$.

\section{RESULTADOS}

Entre dezembro de 2009 e dezembro de 2011, 1.108 pacientes com diagnóstico de IAM foram tratados por ICPp. No total, foram incluídos 680 pacientes no grupo ICPp diurna e 428 no grupo ICPp noturna. Não existiu diferença entre os grupos em relação ao tipo de IAM: tipo 1 (90,1\% vs. 94,8\%), tipo $2(2,7 \%$ vs. $1,4)$, tipo $3(0,1 \%$ vs. 0$)$, e tipo $4 a(0,9 \%$ vs. $0,5 \%)$, respectivamente $(p=0,14)$.
$\mathrm{Na}$ Tabela 1 são apresentadas as características clínicas dos dois grupos, que, em geral, foram semeIhantes. A maioria dos pacientes era do sexo masculino (71\% vs. 66\%; $p=0,09$ ), a média de idades foi de 60 \pm 12 anos vs. $60 \pm 11$ anos $(p=0,89)$, um quinto dos pacientes era diabético $(21,6 \%$ vs. $22,1 \% ; p=0,88)$ e a maioria estava em Killip I (82,6\% vs. $82,7 \%)$. Os pacientes submetidos à ICPp no período noturno, no entanto, apresentaram maior tempo porta-balão (84 \pm 66 minutos vs. $102 \pm 98$ minutos; $p<0,01)$. A Figura 1 apresenta a distribuição do tempo porta-balão em percentis, sendo importante ressaltar que, independentemente do horário da ICPp, o tempo porta-balão foi $<90$ minutos em $75 \%$ dos casos.

As características angiográficas e do procedimento estão detalhadas na Tabela 2. O grupo da ICP noturna apresentava maior número de pacientes com acometi-

TABELA 1

Características clínicas e eletrocardiográficas

\begin{tabular}{|c|c|c|c|}
\hline Variável & $\begin{array}{l}\text { ICPp diurna } \\
(\mathrm{n}=680)\end{array}$ & $\begin{array}{l}\text { ICPp noturna } \\
(\mathrm{n}=428)\end{array}$ & Valor de $p$ \\
\hline Idade, anos & $60 \pm 12$ & $60 \pm 11$ & 0,89 \\
\hline Sexo masculino, \% & 71 & 66 & 0,09 \\
\hline Peso, kg & $76 \pm 13$ & $60 \pm 12$ & 0,68 \\
\hline Altura, cm & $168,3 \pm 8,4$ & $167,1 \pm 8,6$ & 0,30 \\
\hline Tabagismo ativo, \% & 41,2 & 44,6 & 0,23 \\
\hline Hipertensão arterial, \% & 61,4 & 68,2 & 0,02 \\
\hline Diabetes, \% & 21,6 & 22,1 & 0,88 \\
\hline Em uso de insulina & 7,5 & 5,5 & 0,91 \\
\hline Dislipidemia, \% & 32,1 & 36,7 & 0,11 \\
\hline História familiar de DAC, \% & 33,3 & 29,7 & 0,23 \\
\hline ICP prévia, \% & 13,9 & 16,9 & 0,19 \\
\hline CRM prévia, \% & 4,6 & 3,3 & 0,34 \\
\hline IAM prévio, $\%$ & 19,6 & 21,2 & 0,53 \\
\hline Frequência cardíaca, bpm & $78,6 \pm 19,6$ & $79,6 \pm 20,1$ & 0,37 \\
\hline Pressão arterial sistólica, mmHg & $136,1 \pm 72,8$ & $136,4 \pm 29,7$ & 0,93 \\
\hline Pressão arterial diastólica, mmHg & $80,6 \pm 18,4$ & $83,5 \pm 18,1$ & 0,01 \\
\hline Classificação Killip, \% & & & 0,89 \\
\hline 1 & 82,6 & 82,7 & \\
\hline$\|$ & 10,1 & 9,6 & \\
\hline III & 1,3 & 1,9 & \\
\hline IV & 5,9 & 5,8 & \\
\hline \multicolumn{4}{|l|}{ Achados eletrocardiográficos, \% } \\
\hline IAM anterior & 44,1 & 41,1 & 0,35 \\
\hline IAM de ventrículo direito & 10,8 & 10,8 & $>0,99$ \\
\hline BAVT & 1,6 & 4,2 & 0,01 \\
\hline
\end{tabular}

ICPp: intervenção coronária percutânea primária; DAC: doença arterial coronariana; CRM: cirurgia de revascularição miocárdica; IAM: infarto agudo do miocárdio; BAVT: bloqueio atrioventricular total. 
mento de dois ou três vasos $(p<0,01)$, mas os vasos culpados e os fluxos TIMI pré e pós-procedimento

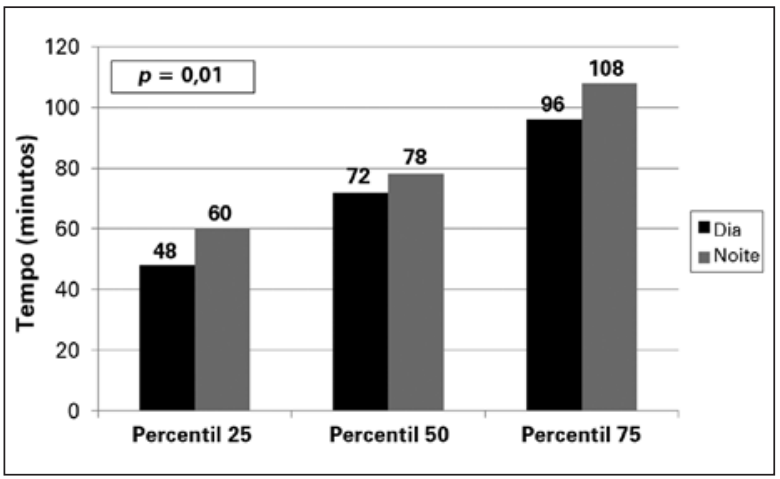

Figura 1. Distribuição do tempo porta-balão por percentil entre os grupos. não foram diferentes entre os grupos. Dispositivos de tromboaspiração foram utilizados em 35\% dos pacientes do grupo diurno e em $38 \%$ dos pacientes do grupo noturno $(p=0,26)$. Uso de balão de contrapulsação aórtica também foi semelhante em ambos os grupos (3\% vs. $2,4 \% ; p=0,70)$.

Em relação à terapia antiplaquetária, verificamos que a dose de ataque de clopidogrel foi semelhante em ambos os grupos: dose de $300 \mathrm{mg}$ (16,3\% vs. 19,2\%; $p=0,21)$ e $600 \mathrm{mg}(81,5 \%$ vs. $80,3 \% ; p=0,69)$. O inibidor de glicoproteína IIb/Illa foi utilizado em $25,8 \%$ dos pacientes do grupo diurno e em 30,2\% dos pacientes do grupo noturno $(p=0,26)$.

A mortalidade no período hospitalar foi de $7,2 \%$ nos pacientes tratados durante o dia e 10,2\% naqueles atendidos durante a noite $(p=0,16)$. Não houve diferença

TABELA 2

Características angiográficas e do procedimento

\begin{tabular}{|c|c|c|c|}
\hline Variável & $\begin{array}{l}\text { ICPp diurna } \\
(\mathrm{n}=680)\end{array}$ & $\begin{array}{l}\text { ICPp noturna } \\
(\mathrm{n}=\mathbf{4 2 8})\end{array}$ & Valor de $p$ \\
\hline Via de acesso, \% & & & 0,49 \\
\hline Femoral & 83,5 & 85,7 & \\
\hline Radial & 16,3 & 14,3 & \\
\hline Extensão da doença arterial coronariana, \% & & & 0,01 \\
\hline 1 vaso & 53,6 & 45,6 & \\
\hline 2 vasos & 28,8 & 35,6 & \\
\hline$\geq 3$ vasos & 17,6 & 18,9 & \\
\hline Lesão de tronco de coronária esquerda, \% & 3,6 & 2,9 & 0,32 \\
\hline Vaso culpado,\% & & & 0,31 \\
\hline DA ou diagonal & 44,4 & 41,4 & \\
\hline CX ou marginal & 12,9 & 14,7 & \\
\hline CD ou ramos & 39,3 & 41,9 & \\
\hline Ramos envolvidos, \% & 13 & 8,4 & 0,47 \\
\hline Calcificação, \% & 15,7 & 17,8 & 0,39 \\
\hline Trombo, \% & 68 & 70,2 & 0,49 \\
\hline Fluxo TIMI pré-procedimento, \% & & & 0,40 \\
\hline 0 e 1 & 70,9 & 75,5 & \\
\hline 2 e 3 & 29,1 & 24,5 & \\
\hline Fluxo TIMI pós-procedimento, \% & & & 0,26 \\
\hline 0 e 1 & 9,4 & 12,9 & \\
\hline 2 e 3 & 90,6 & 87,1 & \\
\hline Pré-dilatação, \% & 63 & 62,8 & 0,98 \\
\hline Pós-dilatação, \% & 23,2 & 27,8 & 0,09 \\
\hline Tromboaspiração, \% & 35 & 38 & 0,26 \\
\hline Diâmetro do stent, mm & $3,16 \pm 0,45$ & $3,15 \pm 0,48$ & 0,02 \\
\hline Comprimento do stent, $\mathrm{mm}$ & $19,3 \pm 6,3$ & $20,4 \pm 6,6$ & 0,06 \\
\hline Pressão de liberação do stent, atm & $14,2 \pm 2,6$ & $14,2 \pm 2,3$ & 0,08 \\
\hline
\end{tabular}


em relação à ocorrência de acidente vascular cerebral $(0,2 \%$ vs. $0,6 \% ; p=0,49)$, trombose de stent $(2,8 \%$ vs. $2,4 \% ; p=0,69)$, sangramento maior $(1,9 \%$ vs. $2,1 \%$; $p=0,50)$, sangramento menor $(7,3 \%$ vs. $7 \% ; p=0,46)$ e necessidade de diálise $(3,7 \%$ vs. $3,9 \% ; p=0,90)$. Não houve diferença estatisticamente significativa em relação à mortalidade em 30 dias entre os pacientes tratados nos períodos diurno ou noturno (Figura 2). Ao final de 1 ano, 97\% dos pacientes apresentaram seguimento clínico disponível. Os preditores de mortalidade em 1 ano foram idade, sexo feminino, diabetes mellitus, e classificação Killip III e IV no momento da admissão hospitalar. ICPp no horário noturno não foi preditor de mortalidade em 1 ano (Tabela 3).

\section{DISCUSSÃO}

Neste estudo, demonstramos que a ICPp realizada no horário noturno não esteve associada a aumento de mortalidade ou de desfechos clínicos adversos. $\mathrm{Na}$ análise multivariada, os preditores de mortalidade em 1 ano foram idade, sexo feminino, diabetes e classificação de Killip, mas não ICPp realizada no horário noturno. Esses dados reforçam a noção e a recomendação de diretrizes de que a ICPp pode estar

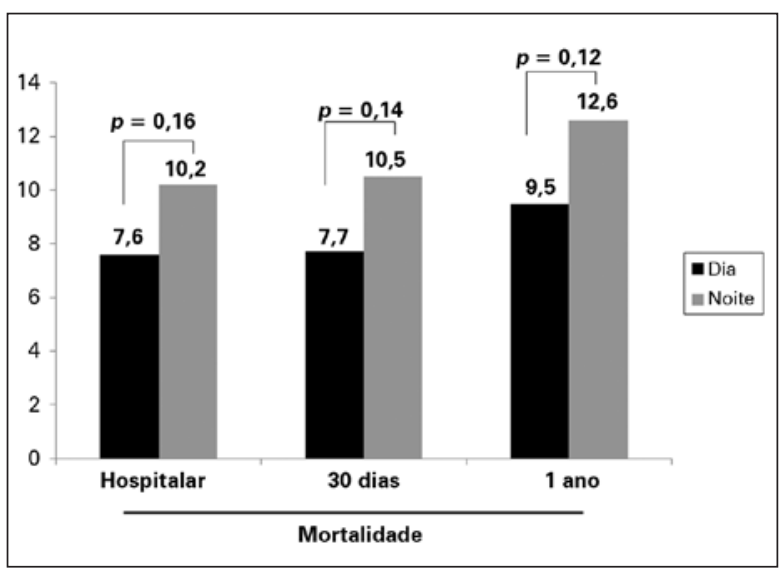

Figura 2. Mortalidade ao longo do tempo de seguimento.

TABELA 3

Análise multivariada dos preditores de mortalidade em 1 ano

\begin{tabular}{lccc}
\hline Variável & $\begin{array}{c}\text { Razão de } \\
\text { chance }\end{array}$ & IC 95\% & $\begin{array}{c}\text { Valor } \\
\text { de } \boldsymbol{p}\end{array}$ \\
\hline Classificação Killip III/IV & 10,02 & $5,8-17,1$ & $<0,01$ \\
Diabetes & 1,7 & $1,11-2,7$ & 0,02 \\
Sexo feminino & 1,7 & $1,13-2,67$ & 0,01 \\
Idade & 1,06 & $1,04-1,08$ & $<0,01$ \\
ICPp noturna & 1,39 & $0,91-2,14$ & 0,12 \\
\hline
\end{tabular}

IC: intervalo de confiança; ICPp: intervenção coronária percutânea primária. disponível durante 24 horas do dia, nos 7 dias da semana. Essa medida é de extrema importância para as políticas de estratégia em saúde, uma vez que cerca de $50 \%$ dos casos de IAM ocorrem fora do horário regular de trabalho. ${ }^{18}$ Por outro lado, esses resultados devem ser avaliados dentro do contexto em que foi realizado este estudo: um centro de referência de alto volume, com operadores com grande experiência e enfermagem de plantão durante as 24 horas do dia no setor de cardiologia invasiva.

Outros estudos têm sugerido que a ICPp pode apresentar resultados diferentes de acordo com o horário realizado. ${ }^{12,13,19}$ Magid et al. ${ }^{14}$ demonstraram que o tempo porta-balão é mais elevado dependendo do horário e do dia da semana em que o evento coronariano ocorre, e nosso estudo corrobora esses achados. O retardo associado à $\mathrm{ICPp}$ realizada no período noturno foi de 18 minutos. Acreditamos que esse atraso deva-se à complexidade do procedimento, que envolve uma equipe maior comparada à administração de trombolítico. Apesar dessa pequena demora, observamos que menos de $25 \%$ da amostra, independentemente do horário, ultrapassou os 90 minutos preconizado pelas diretrizes. ${ }^{7}$

Embora o tempo porta-balão tenha sido discretamente elevado no grupo submetido à ICPp no período noturno, não foi observada diferença estatisticamente significativa em relação aos desfechos hospitalares e à mortalidade em 1 ano entre os grupos. Em recente estudo observacional, foi demonstrado, pelo National Cardiovascular Data Registry ${ }^{\circledR}\left(\mathrm{NCDR}^{\circledR}\right),{ }^{20}$ que a mortalidade cardiovascular foi reduzida nos últimos anos entre os pacientes com IAM para cerca de 5\%. Já na literatura nacional, Piegas e Haddad ${ }^{21}$ reportaram taxa de óbito relacionada ao infarto de 6,35\%. Em nosso estudo, a mortalidade hospitalar diurna foi $7,6 \%$ e a noturna de 10,2\%. Embora não tenha ocorrido significância estatística, uma possível explicação para a maior mortalidade noturna seria a maior gravidade dos pacientes com atendimento durante a noite, uma vez que apresentaram maior prevalência de bloqueio atrioventricular total e comprometimento coronariano mais severo. Além disso, o retardo no tempo porta-balão pode ter desempenhado papel importante nos desfechos clínicos observados.

\section{Limitações do estudo}

Nosso estudo apresenta algumas limitações. A inclusão de pacientes provenientes de um único centro pode limitar a extrapolação destes dados para outros hospitais, principalmente aqueles de baixo volume e que não dispõem de plantão da equipe da cardiologia invasiva por 24 horas. Por outro lado, essa análise poderia estimular outros estudos em nosso meio para avaliar a realidade da ICPp no Brasil, já que este é o principal método de reperfusão utilizado atualmente. 
Este estudo apresentou uma das maiores casuísticas de ICPp já relatadas em nosso meio, mas, pela ocorrência relativamente infrequente dos desfechos, o número de pacientes pode ter sido insuficiente para identificar diferenças significativas.

\section{CONCLUSÕES}

A realização de intervenção coronária percutânea primária no horário noturno não foi associada com aumento de eventos cardiovasculares adversos e sangramentos, mas os pacientes apresentaram tempo porta-balão significativamente mais elevado do que aqueles tratados no horário diurno. Esses resultados reforçam a segurança da intervenção coronária percutânea primária realizada em regime de plantão, conforme demonstrado em ensaios clínicos randomizados e recomendado pelas diretrizes atuais. Além disso, devem estimular esforços na tentativa de diminuir o tempo porta-balão em pacientes atendidos no período noturno.

\section{CONFLITO DE INTERESSES}

Não há.

\section{FONTE DE FINANCIAMENTO}

Não há.

\section{REFERÊNCIAS}

1. Gruppo Italiano per lo Studio della Streptochinasi nellInfarto Miocardico (GISSI). Effectiveness of intravenous thrombolytic treatment in acute myocardial infarction. Lancet. 1986;1(8478): 397-402.

2. Randomised trial of intravenous streptokinase, oral aspirin, both, or neither among 17,187 cases of suspected acute myocardial infarction: ISIS-2. ISIS-2 (Second International Study of Infarct Survival) Collaborative Group. Lancet. 1988; 2(8607):349-60.

3. O'Neill W, Timmis GC, Bourdillon PD, Lai P, Ganghadarhan $\mathrm{V}$, Walton J Jr., et al. A prospective randomized clinical trial of intracoronary streptokinase versus coronary angioplasty for acute myocardial infarction. N Engl J Med. 1986;314(13):812-8.

4. Andersen HR, Nielsen TT, Rasmussen K, Thuesen L, Kelbaek $\mathrm{H}$, Thayssen $\mathrm{P}$, et al. A comparison of coronary angioplasty with fibrinolytic therapy in acute myocardial infarction. $N$ Engl J Med. 2003;349(8):733-42.

5. Grines C, Patel A, Zijlstra F, Weaver WD, Granger C, Simes RJ. Primary coronary angioplasty compared with intravenous thrombolytic therapy for acute myocardial infarction: sixmonth follow up and analysis of individual patient data from randomized trials. Am Heart J. 2003;145(1):47-57.

6. Keeley EC, Boura JA, Grines CL. Primary angioplasty versus intravenous thrombolytic therapy for acute myocardial infarction: a quantitative review of 23 randomised trials. Lancet. 2003; 361(9351):13-20.

7. Mattos LA, Lemos Neto PA, Rassi Junior A, Marin-Neto JA, Souza AGMR, Devito FS. Diretrizes da Sociedade Brasileira de Cardiologia - Intervenção Coronária Percutânea e Métodos Adjuntos Diagnósticos em Cardiologia Intervencionista. Rev Bras Cardiol Invasiva. 2008;16 Supl. 2:9-88.

8. Nielsen $\mathrm{PH}$, Maeng M, Busk M, Mortensen LS, Kristensen SD, Nielsen TT, et al. Primary angioplasty versus fibrinolysis in acute myocardial infarction: long-term follow-up in the Danish acute myocardial infarction 2 trial. Circulation. 2010; 121(13):1484-91.

9. Zijlstra F, de Boer MJ, Hoorntje JC, Reiffers S, Reiber JH, Suryapranata H. A comparison of immediate coronary angioplasty with intravenous streptokinase in acute myocardial infarction. N Engl J Med. 1993;328(10):680-4.

10. Antman EM, Anbe DT, Armstrong PW, Bates ER, Green LA, Hand $M$, et al. ACC/AHA guidelines for the management of patients with ST-elevation myocardial infarction--executive summary: a report of the American College of Cardiology/ American Heart Association Task Force on Practice Guidelines (Writing Committee to Revise the 1999 Guidelines for the Management of Patients With Acute Myocardial Infarction). Circulation. 2004;110(5):588-636.

11. Kushner FG, Hand M, Smith SC Jr, King SB 3rd, Anderson JL, Antman EM, et al. 2009 Focused Updates: ACC/AHA Guidelines for the Management of Patients With ST-Elevation Myocardial Infarction (updating the 2004 Guideline and 2007 Focused Update) and ACC/AHA/SCAI Guidelines on Percutaneous Coronary Intervention (updating the 2005 Guideline and 2007 Focused Update): a report of the American College of Cardiology Foundation/American Heart Association Task Force on Practice Guidelines. Circulation. 2009;120(22):2271-306.

12. De Luca G, Suryapranata H, Ottervanger JP, van't Hof AW, Hoorntje JC, Gosselink AT, et al. Circadian variation in myocardial perfusion and mortality in patients with ST-segment elevation myocardial infarction treated by primary angioplasty. Am Heart J. 2005;150(6):1185-9.

13. Henriques JP, Haasdijk AP, Zijlstra F. Outcome of primary angioplasty for acute myocardial infarction during routine duty hours versus during off-hours. J Am Coll Cardiol. 2003; 41(12):2138-42.

14. Magid DJ, Wang Y, Herrin J, McNamara RL, Bradley EH, Curtis JP, et al. Relationship between time of day, day of week, timeliness of reperfusion, and in-hospital mortality for patients with acute ST-segment elevation myocardial infarction. JAMA. 2005;294(7):803-12.

15. Saleem MA, Kannam H, Aronow WS, Weiss MB, Kalapatapu $\mathrm{K}$, Pucillo AL, et al. The effects of off-normal hours, age, and gender for coronary angioplasty on hospital mortality in patients undergoing coronary angioplasty for acute myocardial infarction. Am J Cardiol. 2004;93(6):763-4.

16. Cardoso CO, Quadros AS, Voltolini I, Azmus AD, Cardoso CR, Sebben JC, et al. Angioplastia primária no infarto agudo do miocárdio: existe diferença de resultados entre as angioplastias realizadas dentro e fora do horário de rotina? Rev Bras Cardiol Invasiva. 2011;18(3):273-80.

17. Thygesen K, Alpert JS, White HD, Jaffe AS, Apple FS, Galvani $M$, et al. Universal definition of myocardial infarction. Circulation. 2007;116(22):2634-53.

18. Uyarel H, Ergelen M, Akkaya E, Ayhan E, Demirci D, Gul $M$, et al. Impact of day versus night as intervention time on the outcomes of primary angioplasty for acute myocardial infarction. Catheter Cardiovasc Interv. 2009;74(6):826-34.

19. Kostis WJ, Demissie K, Marcella SW, Shao YH, Wilson AC, Moreyra AE; Myocardial Infarction Data Acquisition System (MIDAS 10) Study Group. Weekend versus weekday admission and mortality from myocardial infarction. N Engl J Med. 2007;356(11):1099-109.

20. Roe MT, Messenger JC, Weintraub WS, Cannon CP, Fonarow GC, Dai D, et al. Treatments, trends, and outcomes of acute myocardial infarction and percutaneous coronary intervention. J Am Coll Cardiol. 2010;56(4):254-63.

21. Piegas LS, Haddad N. Percutaneous coronary intervention in Brazil: results from the Brazilian Public Health System. Arq Bras Cardiol. 2011;96(4):317-24. 